\title{
PID CLOSED-LOOP CONTROL SYSTEM BASED ON S7-300 PLC
}

\author{
LI Xin ${ }^{1}$, LEI Ju-yang ${ }^{2}$ \\ ${ }^{I}$ College of Mechanical Engineering, Shanghai University of Engineering Science, Shanghai, China \\ ${ }^{2}$ College of Mechanical Engineering, Shanghai University of Engineering Science, Shanghai, China
}

\begin{abstract}
In order to eliminate and reduce the error between the output variable and the given value in the automatic control system, the dynamic performance and static performance of the system meet the requirements and the system can run stably. Through parameter setting method, the structure and parameters of PID controller are set and PID closed-loop control was simulated by using S7-300plcsim, so as to adjust the proportion, integral and differential links of PID control system.
\end{abstract}

Keywords: -Automatic control system; Parameter setting; PID controller; S7-300

$* * *$

\section{INTRODUCTION}

In industrial production, closed-loop control is generally used to control a series of continuously changing analog quantities such as temperature, pressure, and flow[1].Closed-loop control system of simple structure, easy to realize automatic control, so it has been widely used in various fields, and the PID controller is the most widely used the closed loop control system, PID is the abbreviation of proportion, integral and differential.

\section{STRUCTURE AND PARAMETERS OF PID} CONTROLLER

S7-300 has a dedicated closed-loop control module, generally using ordinary signal modules and dedicated function modules to achieve PID control[ ].You can select FB41 FB43 and FB58 and FB59 in the "\ LibrarylStandard Library\PID Controller" of the left window of the program editor, where the digital value output of FB41 is generally converted to continuous analog quantity by AO module. The FB41 can be used as a standalone PID controller in some cases or it can be expanded with the pulse generator FB43 to generate pulse width modulated switching output signals to control the proportional actuator[2].

The output expression of the analog PID controller is

$$
m v(t)=K_{p}\left[e v(t)+\frac{1}{T_{1}} \int e v(t) d t+T_{D} \frac{d e v(t)}{d t}\right]+M
$$

In the formula, the error signal is

$$
e v(t)=s p(t)-p v(t)
$$

$\operatorname{sp}(t)$ is the set value, $p v(t)$ is the feedback value, $\operatorname{mv}(t)$ is the output signal of the controller, $\mathrm{Kp}$ is the proportional coefficient,Ti and TD is the integral time and differential time respectively, $\mathrm{M}$ is the initial value of the integral part[ $3]$.

\section{PID CONTROLLER PARAMETER SETTING}

In order to make the system dynamic and static performance meet the requirements, so that the system can run steady, need to set the parameters of the controller, using PID control to the main parameters setting are proportional GAIN, integral GAIN time TI, differential time TD and sampling time CYCLE[4].

\subsection{Parameter Setting Method}

Setting of PID parameters is an integrated, the process of parameter influence each other, and setting the PID controller parameters can be through the following two ways, one is the PID parameters of S7-300 integrated assignment tool, according to the PID controller of square wave given curve and was accused of amount of step response curve, observe the closed loop control effect. The second is to use the PID parameter assignment tool to modify the parameters of the PID controller and to adjust the PID controller parameters by observing the relationship between the PID controller parameters and the system performance. In order to reduce the amount to be adjusted, the PI controller can be limited, input a step given signal to the system, observe the waveform of the process variable , and obtain the information of system performance. If the overshoot of step response is too large and the period of entering steady state is too long or always oscillating, the integral time $T_{1}$ should be increased or the gain $K_{P}$ of controller should be reduced. If the step response has no 
overshoot, but the controlled amount rises too slowly and the transition time is long, the parameters should be adjusted by decreasing the integral time or increasing the gain of the controller. If the speed of eliminating the error is too slow, the integration time should be appropriately reduced to enhance the integral action. Repeatedly adjusting the gain and integration time, if the overshoot is still large, you can add a derivative action, that is, using PID control, the derivative time TD gradually increases from 0 , repeatedly adjusts $K_{P}, T_{I}$ and $T_{D}$ until it meets the requirements. Note that when adjusting the value of the gain, the value of the integral component and the derivative component is affected at the same time, rather than only affecting the proportional component. If the actual curve takes a long time to reach the steady state value for the first time, the gain value should be increased. If the overshoot is also increased, the gain $K_{P}$ can be compensated by increasing $T_{I}$ and $T_{D}$ to adjust [5].

\subsection{Determination of Sampling Time}

The PID control program is executed periodically, and the execution period is called sampling time $\boldsymbol{T}_{S}$. The smaller the sampling time is, the more the sampling value can simulate the change of quantity. However, the smaller the sampling time is, it will increase the calculation amount of CPU, so the sampling time should not be too small. Determine the sampling time, shall ensure that the accused is rapidly changing to have enough sample points, in order to make sure won't be lost because of sampling point is too thin and analog information collected.

\subsection{The Initial Parameter Value of the PID}

\section{Controller}

In order to ensure the safety of the system, avoid the instability occurs when system is first put into operation or the abnormal situation of the large amount of overshoot, in the first set when the parameters of the more conservative, the gain does not want too big, integration time should not be too small. In addition, it is necessary to formulate the emergency treatment plan of the controlled volume response curve which rises too fast and may lead to large overshoot, after the test run, according to the waveform of the response curve, the system performance information can be obtained, modify the parameters of the controller according to the rule of adjusting PID controller parameters.

\section{SIMULATION EXPERIMENT OF PID CONTROLLER PARAMETER TUNING}

\subsection{Establish PID Control Project}

Create a project called "PIDControl" for the s7-300 project, add SIMATIC 300 Station,add the power module PS 307 5A and sm-300 modules, go under the block, and right click the mouse, insert "Variable Table" as is shown in Figure1,In this page insert two organization blocks OB35 and OB100,insert function block FB41 and FB100,insert function SFB41 and two data blocks DB41 and DB100.

\begin{tabular}{|c|c|c|c|c|c|c|}
\hline \multicolumn{7}{|c|}{ 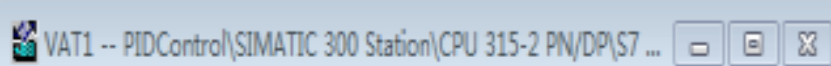 } \\
\hline & A Adidress & Sratol & Display fornat & Status valua & Wodify & $\therefore$ \\
\hline 1 & DEA1.DED 20 & PID_DI" & FLOLTTH_POIII & & & \\
\hline 2 & DBA1.DED 24 & PID_DI' & IIIE & & & $\equiv$ \\
\hline 3 & DBA1.DED 28 & PID_DI" & IIIE & & & \\
\hline 4 & DBA1.DED 6 & PID_DI' & FLOLTIN, POIBI & & & \\
\hline 5 & DB41.DED 10 & PID_DI" & FLOATH_ POTII & & & \\
\hline 6 & DBA1.DED 72 & PID_DI" & FLOATTIS_POIDI & & & \\
\hline 7 & DBA1.JED 96 & PID_DI" & FLOATTI__POIII & & & . \\
\hline 1 & & 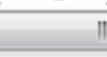 & & & 1 & \\
\hline
\end{tabular}

Fig 1: Variable Table

In OB1, an oscillation circuit is formed using T8 and T9, and the normally open contact of T8 is turned on and off for 30 seconds. "PID_DI".SP_INT is the set value of SP_INT for the controller floating point number in percentage in the background data block PID_DI of FB41. When the normally open contact of T8 is just connected and just broken, the set value SP_INT is changed to floating point number $20.0 \%$ and $70.0 \%$ respectively, and the set value is square wave, OB1 ladder diagram as shown in Figure 2. In OB100, call FB41 and the function block FB100 that simulates the controlled object. Set the initial value of the following input parameters.Make the start flag COM_RST of FB41 and FB100 TRUE, initialize the internal parameters of the PID controller and the controlled object to default values, The sampling time is set to $200 \mathrm{~ms}$, Can also be several times the cycle execution cycle of OB35, Set the controlled object's gain GAIN to 3.0, the time constants of the three inertial links are $5 \mathrm{~s}, 2 \mathrm{~s}$ and 0 s respectively, set the initial values of GAIN, TI and TD of PID controller parameters. The default setting of FB41 is PI controller, set the parameter D_SEL to 1 , the controller is a PID controller, set MAN_ON to FALSE. The controller works in automatic mode,before exiting OB100. Reset the starting token COM_RST of the two FB bits, when FB41 and FB100 are executed later, the value of COM_RST bits is 0 . 


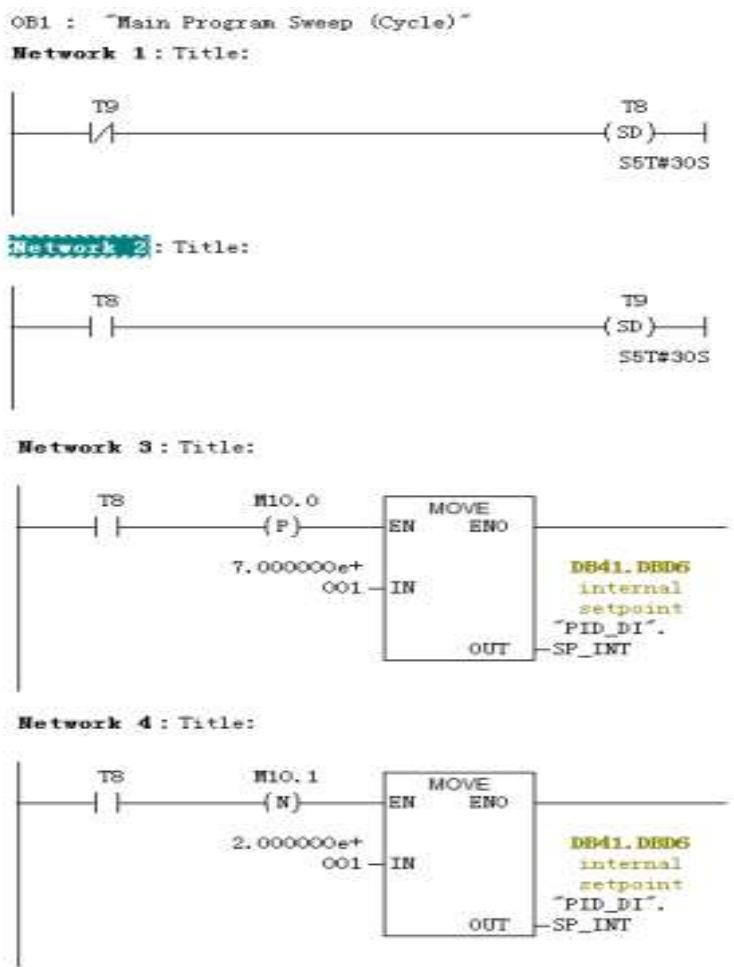

In order to ensure the accuracy of the sampling time of PID operation, FB41 and FB100 are called in the circular interrupt tissue block OB35. In OB35, use the initial parameter values of FB41 and FB100 set in OB100, knowing that the PID parameter assignment tool modifies some of these parameters.

\subsection{PID Controller Parameter Setting Process}

Open the PLCSIM and load all blocks into the simulate PLC, and click the RUN-UP mode.Then click the start button in the lower left corner and execute menu command "START" to "ALL PROGRAMS" to "Siemens Automation"to "SIMATIC"to "STEP 7"to "PID Control Parameter Assignment", open the view of "PIDControl",click the Browser button and,choice the program of "PIDControl"and click the online button,select the background data block DB41 of the FB41 in this project. The parameter assignment dialog box shown in Figure 3 appears to modify the parameters of the PID controller.

Fig 2: OB1 ladder diagram

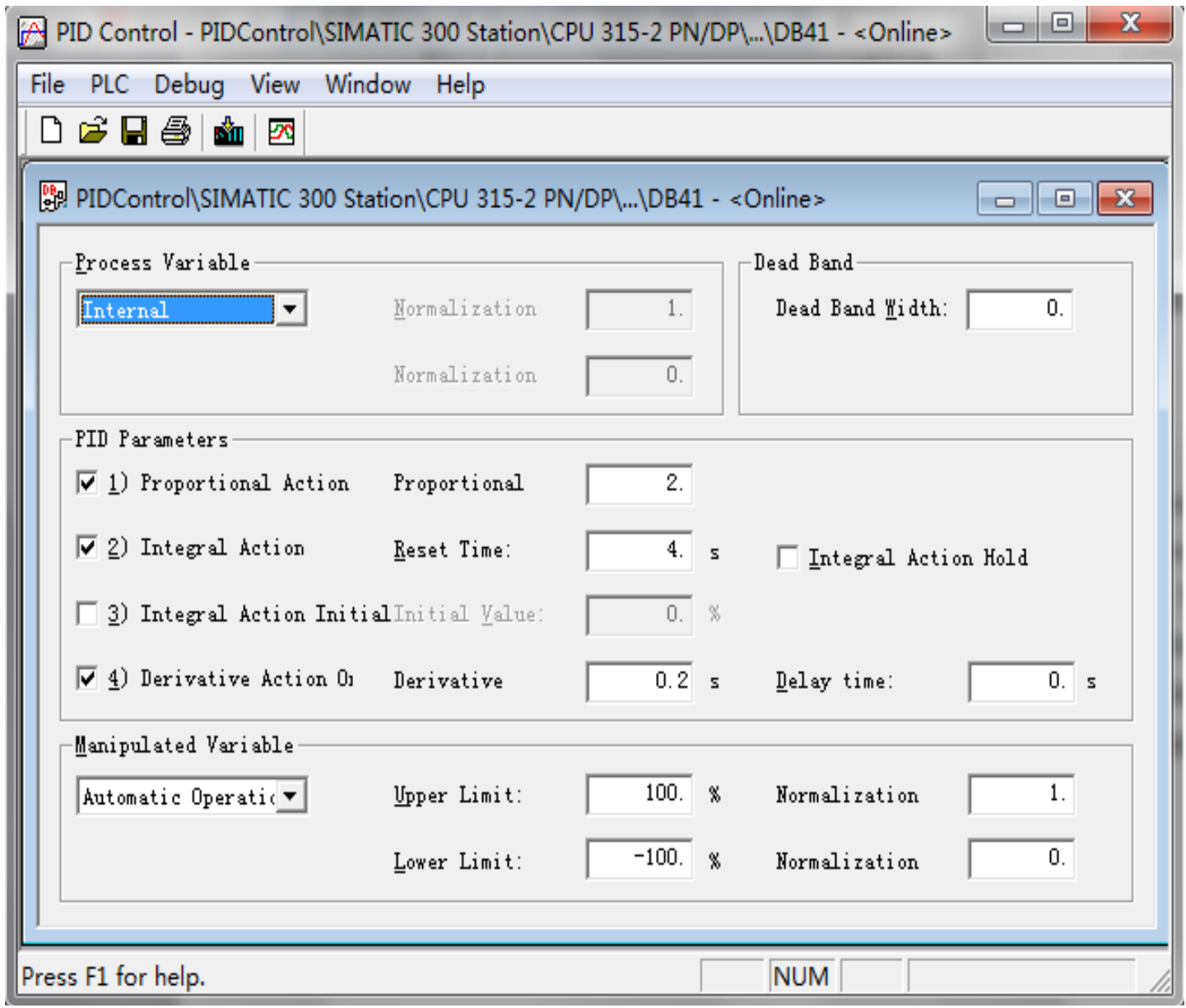

Fig 3: The parameter assignment dialog box 
Click the Curve Record button on the toolbar to open the Settings dialog box, change the curve 3 from "Manipulated" to" None"and only show the curve of "Setpoint Value"and "ProcessValue". Set each curve the Y Axis Limit to 0 and Length of time to 80s, as is shown in Figure 4,

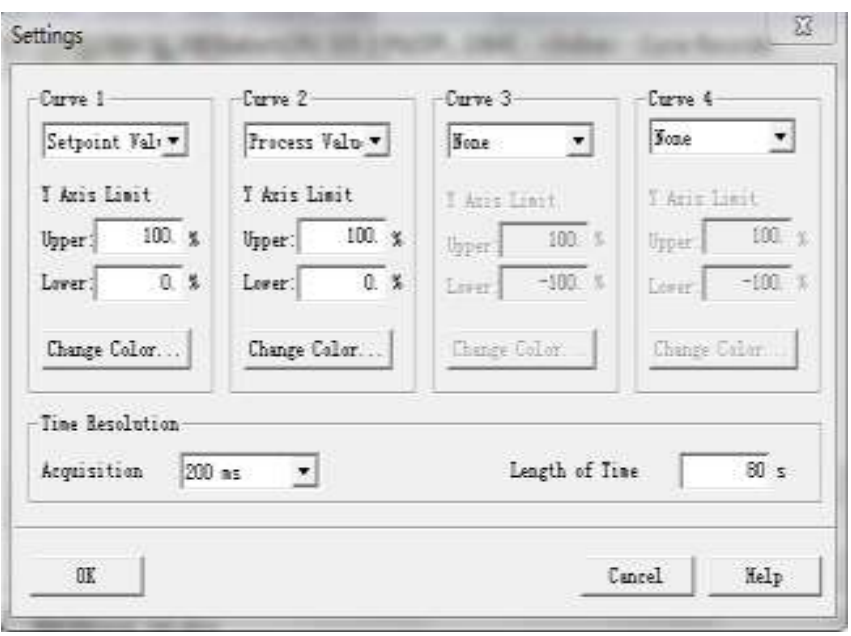

Fig 4: The settings of curves

Click the "Start" button and observe real-time curve shown in Figure 5,

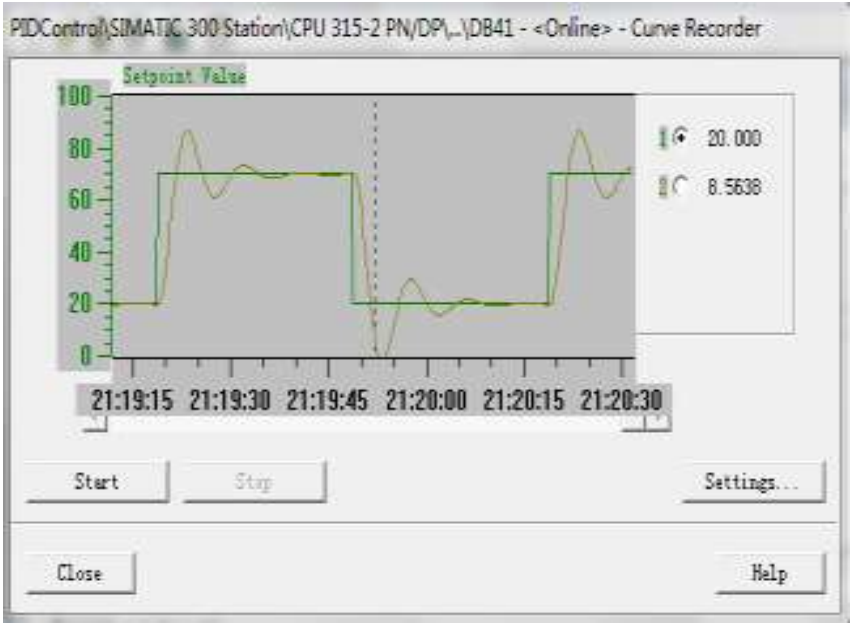

Fig 5: PID control step response curve

As can be seen from Figure 5, the overshoot of the controlled curve is too large and it oscillates several times.Change the integration time from $4 \mathrm{~s}$ to 8 s, download the modified parameters to the simulation PLC, and observe the image as shown in Figure 6.

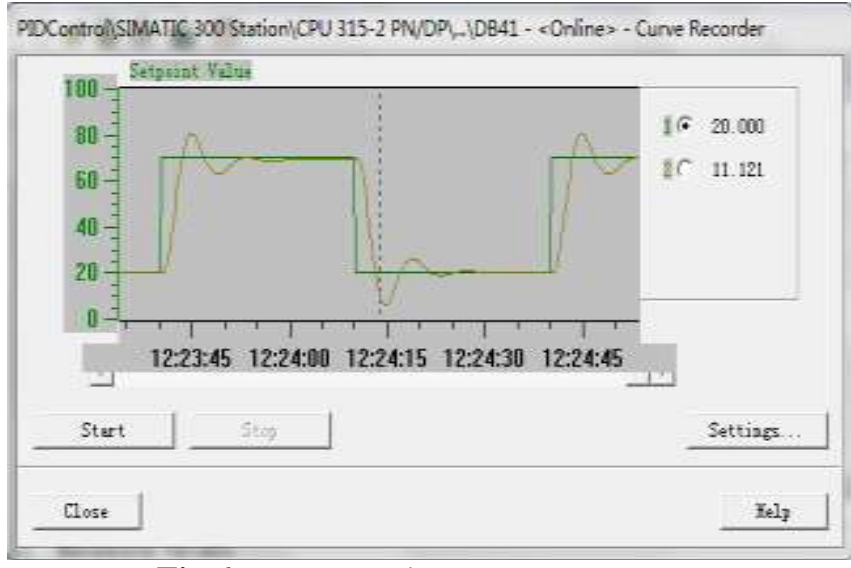

Fig 6: PID control step response curve

As can be seen from figure 6 that the overshoot of the controlled curve decreases significantly and the number of oscillations still exists.Change the differential time from $0.2 \mathrm{~s}$ to $1.0 \mathrm{~s}$, and download the modified parameters to PLC, as shown in figure 7 .

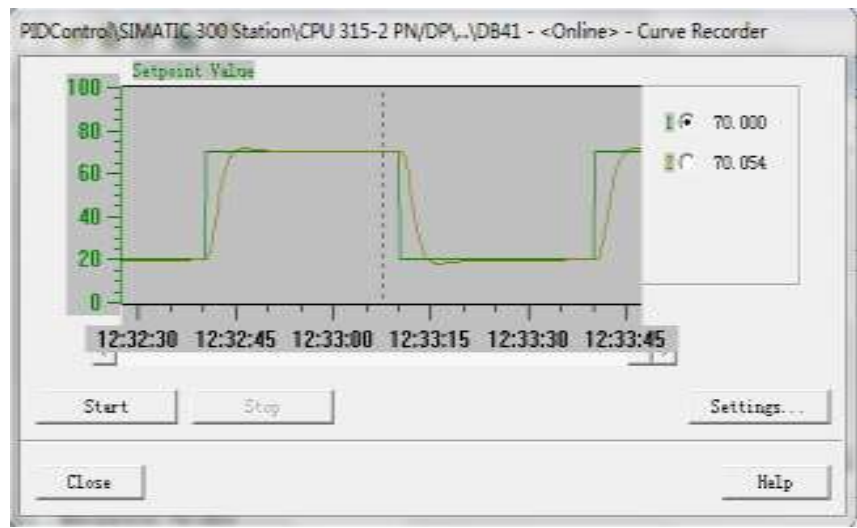

Fig 7: PID control step response curve

In figure 7 , the number of oscillations is significantly reduced. In order to speed up the elimination of error, the integration time is reduced to $6 \mathrm{~s}$. As shown in figure 8 , when , , the overshoot, the rise time and the speed of eliminating error reach the ideal state.

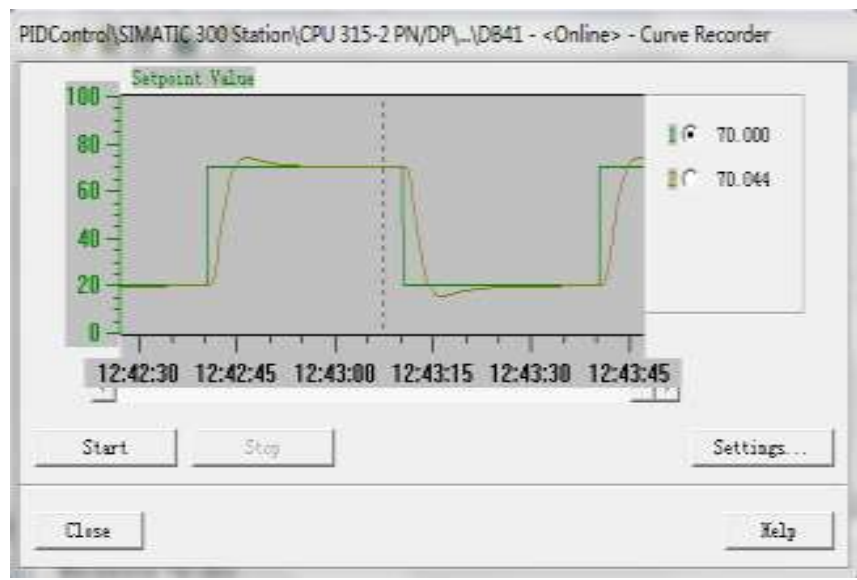

Fig 8: PID control step response curve 


\section{CONCLUSION}

PID control adopts completely different control ideas and it does not need to use the mathematical model of the controlled object, the ideal control effect can be obtained by adjusting a small number of parameters of the controller. The structure of PID controller is simple and easy to realize and PLC manufacturer provides a variety of hardware and software products to realize PID control function. The PID controller can have more flexibility and adaptability in implementing complex control such as multi-loop control and cascade control.

\section{REFERENCES}

[1] Zhang Jie,Li Bo.Application of Siemens PLC in Air Drying Station[J].Inner Mongulia Petrochemical Industry, 2015,41(17):37-38.

[2] Liao Changchu, followed me hands-on learning S7300/400PLC [M]. Machinery Industry Press, Beijing, 2017, 206-223.

[3] Zhang Fuqiang. Graphical method for designing stability margin of PID controller with time-delay system[D]. Tianjin University of Science and Technology, 2013.

[4] Huang S. Safety. Design and implementation of negative pressure control system based on IPC[D]. Hefei University of Technology, 2017.

[5] Liao Changchu, S7-300/400 Applied Technology [M]. Mechanical Industry Press, Beijing, 2016, 377381. 